\title{
Transistors based on the Guanosine molecule (a DNA base)
}

\author{
S. D’Amico ${ }^{\mathrm{a}, *}$, G. Maruccio ${ }^{\mathrm{a}}$, P. Visconti ${ }^{\mathrm{a}}$, E. D’Amone $^{\mathrm{a}}$, R. Cingolani $^{\mathrm{a}}$, \\ R. Rinaldi ${ }^{\mathrm{a}}$, S. Masiero ${ }^{\mathrm{b}}$, G.P. Spada ${ }^{\mathrm{b}}$, G. Gottarelli $^{\mathrm{b}}$ \\ ${ }^{a}$ National Nanotechnology Laboratory of INFM, University of Lecce, 73100 Lecce, Italy \\ ${ }^{\mathrm{b}}$ Department of Organic chemistry, University of Bologna, 40100 Bologna, Italy
}

Received 7 May 2003; revised 22 June 2003; accepted 27 June 2003

\begin{abstract}
Molecules are attractive to develop nano-electronic devices. In this paper a new type of transistor is realized by using self-organized films of the Guanosine molecule, a modified DNA base. With its $40 \mathrm{~nm}$ channel length the transistor is a good starting point for a new class of nano-electronics devices.

Experimental current-voltage characteristics are shown. A circuital model is also proposed.

(C) 2003 Elsevier Ltd. All rights reserved.
\end{abstract}

Keywords: Molecular Electronics; Transistor; Nanotechnology

\section{Introduction}

Silicon-based microelectronics is reaching a level of miniaturization such that tunneling cannot be avoided and the control of doping in ultra small regions becomes problematic [1,2]. Though it is likely that silicon-based technology will continue to drive the electronic market by virtue of the existing vast infrastructure and manufacturing capabilities, novel and alternative approaches [3] may give new insights and ultimately may usher a new era in nanoelectronics. Molecules [4,5] as individual active devices are appealing candidates for the ultimate miniaturization of devices at the nanoscale.

In this work, a $40 \mathrm{~nm}$ channel length three-terminal electronic device based on deoxyguanosine derivatives (a DNA base) is demonstrated. Systematic transport experiments have been performed to test the influence of the gate-voltage on the current flowing through the source and drain electrodes interconnected by the DNA base. The obtained results suggest that these devices behave like p-channel MOSFETs. Such a transistor-like device represents an excellent starting point towards the development of planar solid-state molecular electronic devices. This

\footnotetext{
* Corresponding author. Tel.: +390-8323-25362; fax: + 39-0832297352.

E-mail address: stefano.damico@unile.it (S. D’Amico).
}

paper is organized as follows: in Section 2 the device structure is described, in Section 3 the experimental results are discussed and the circuital model of the device is presented. Section 4 concludes the paper.

\section{Devices structure}

The presented nanodevices were fabricated starting from a deoxyguanosine derivative $\left(\mathrm{dG}\left(\mathrm{C}_{10}\right)_{2}\right)$, whose molecular structure is shown in Fig. 1. The Guanosine has been chosen because of its peculiar sequence of $\mathrm{H}$ bonds donor or acceptor groups and because of the lowest oxidation potential among the DNA bases, which favour self-assembly and carriers transport, respectively. Previous work [6,7] demonstrated the arrangement of these molecules in ordered, periodic structures and the correlation between supramolecular ordering and conduction properties by means of combined Atomic Force Microscope (AFM) and transport studies. Such Guanosine structures take the form of long ribbons, as shown in Fig. 1, with a strong intrinsic dipole moment along the ribbon axis which causes current rectification in transport experiments [7]. Fig. 2 shows the device structure. The prototype structure investigated here is a planar metalinsulator-metal nanojunction, consisting of two arrowshaped metallic electrodes connected by the molecular 


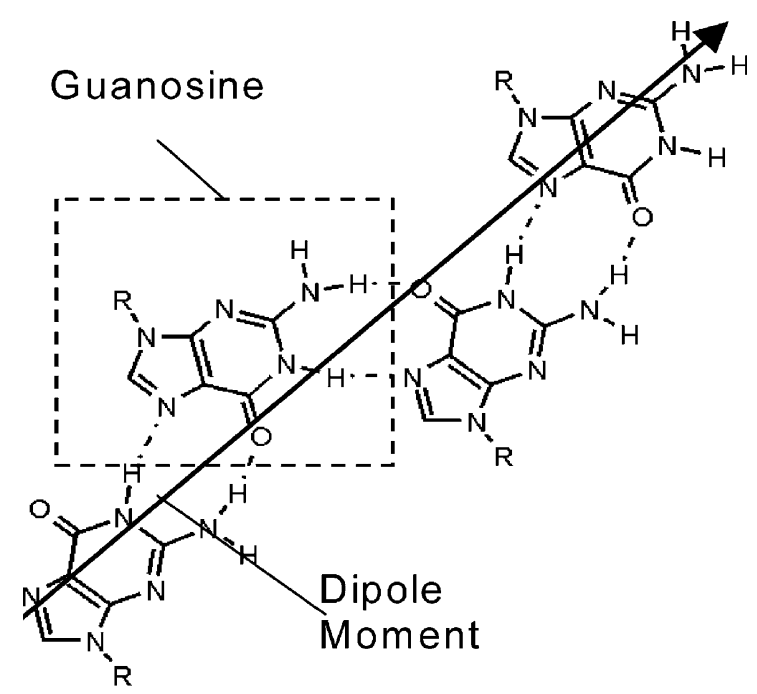

Fig. 1. Guanosine ribbon: the Guanosine molecule and the dipole moments are indicated.

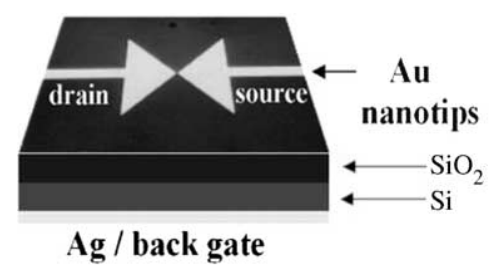

Fig. 2. Device structure.

ribbons. The distance between the electrodes is $40 \mathrm{~nm}$. The electrodes are fabricated by electron beam lithography and lift-off onto a $\mathrm{Si} / \mathrm{SiO}_{2}$ substrate. The thickness of $\mathrm{SiO}_{2}$ is $100 \mathrm{~nm}$. A third (Ag) electrode, the gate, is deposited on the back of the device. The molecules are introduced through the deposition of a drop of a Guanosine solution between the electrodes upon controlled conditions. The evaporation of the organic solvent allows the self-assembly of the molecules on the substrate, resulting in the formation of a thin periodic film of Guanosine ribbons with excellent conductive properties.

\section{Electrical properties}

Fig. 3 shows the measured drain-source current, $I_{\mathrm{ds}}$, versus the drain-source voltage, $V_{\mathrm{ds}}$, as the gate voltage, $V_{\mathrm{g}}$, is varied from 0 to $1 \mathrm{~V}$. In each curve, $I_{\mathrm{ds}}$ shows a clear onset corresponding to a threshold $V_{\mathrm{ds}}$, above which $V_{\mathrm{ds}}, I_{\mathrm{ds}}$ grows exponentially. The $I_{\mathrm{ds}}-V_{\mathrm{ds}}$ curves are found to shift with increasing $V_{\mathrm{g}}$. By keeping constant $V_{\mathrm{ds}}, I_{\mathrm{ds}}$ decreases when $V_{\mathrm{g}}$ increases like in a p-type metal-oxide semiconductor field-effect transistor. The max applied $V_{\mathrm{ds}}$ is limited to $3.5 \mathrm{~V}$ to avoid damage of the device [8].

AFM studies indicate that the device conduction depends on the molecules arrangement on the substrate

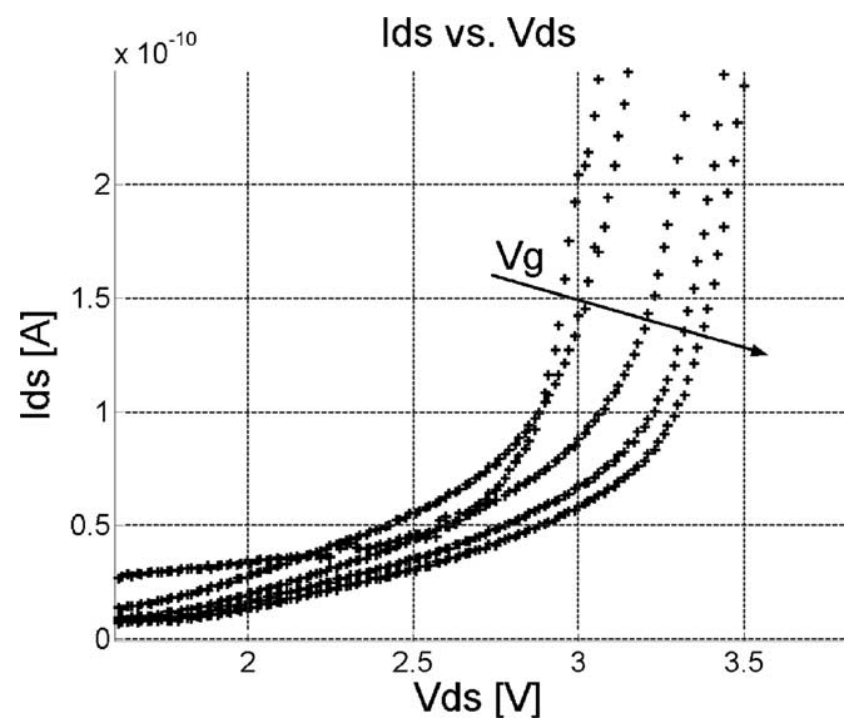

Fig. 3. $I_{\mathrm{ds}}$ versus $V_{\mathrm{ds}}$ experimental curves.

[7]. It has been observed that the alignment of the dipoles associated to the ribbons always results in a macroscopic polarity set into the device. Therefore, the molecular layer can be treated, as first approximation, as compared to a $\mathrm{p}-\mathrm{n}$ junction where the current increases exponentially with $V_{\mathrm{ds}}$. $I_{\mathrm{ds}}$ consists of the current passing through the molecules and the current passing through the $\mathrm{SiO}_{2}$ substrate. The molecular layer conducts when $V_{\mathrm{ds}}$ overcomes the breakdown voltage of the potential barrier at the interfaces with the Au contacts. $V_{\mathrm{g}}$ modifies the electric field configuration into the device changing the breakdown voltage. This allows a modulation of the $I_{\mathrm{ds}}$ by the gate electrodes.

Fig. 4 shows the equivalent circuit of the device. The junction's barriers are schematised as inverse biased diodes. The measured break down voltage, $V_{b}$, is $2.8 \mathrm{~V}$ at $V_{\mathrm{g}}=0$. The $\mathrm{SiO}_{2}$ substrate is represented as a resistance $R_{\mathrm{S}}$ between the source and drain nodes whose measured value is about $50 \mathrm{G} \Omega$.

The current source represents the current flowing though the molecules. In an analogy with the $\mathrm{p}-\mathrm{n}$ junction, the expression of the current source is:

$$
I_{0}=I_{\mathrm{s}}\left(\exp \left(\frac{V_{\mathrm{ds}}-V_{\mathrm{b}}-\alpha V_{\mathrm{g}}}{\eta V_{\mathrm{th}}}\right)-1\right)
$$

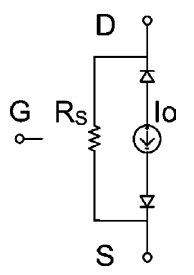

Fig. 4. Equivalent circuit of the device. 


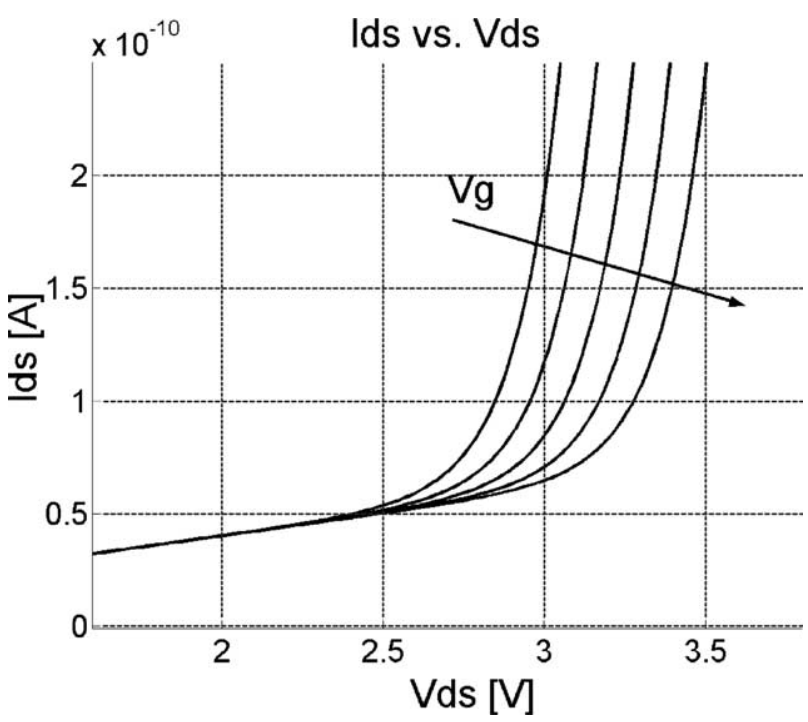

Fig. $5 . I_{\mathrm{ds}}$ vs. $V_{\mathrm{ds}}$ simulated curves.

where $I_{\mathrm{s}}(=7 \mathrm{pA}$, experimentally determined $)$ is the inverse saturation current, $V_{\mathrm{th}}$ is the thermal voltage, $V_{\mathrm{b}}$ (=2.8V@ $V_{\mathrm{g}}=0$, experimentally determined) is the breakdown voltage, $\alpha(=0.5$, experimentally determined) takes into account the effect of the breakdown voltage modulation by $V_{\mathrm{g}}$, while $\eta(=5)$ is an empirical dimensionless constant.

Fig. 5 shows the $I_{\mathrm{ds}}$ vs. $V_{\mathrm{ds}}$ curves obtained by simulating the electric behaviour of the device with the above circuital model. The small signal gain is about 0.5 and it is comparable to the one of the carbon nanotube transistors [5].

\section{Conclusions}

A transistors with a $40 \mathrm{~nm}$ channel length has been realized. The transistor is a good starting point for a new class of sub-micrometer electronic devices. The transistor is based on the Guanosine molecule.

The device structure has been described and the experimental curves have been shown. An attempt to explain the conduction mechanisms has be made and a circuital model has been proposed.

\section{References}

[1] Hiroshi Iwai CMOS Technology-Year 2010 and Beyond, IEEE Journal of Solid State Circuits 34 (3) (1999) 357-366.

[2] M. Yoshizawa, S. Moriya, Resolution limiting mechanism in electron beam lithography, Electronics Letters 36 (1) (2000).

[3] C. Joachim, J.K. Gimzewski, A. Aviram, Electronics using hybridmolecular and mono-molecular devices, Nature 408 (2000) 541-548.

[4] M.A. Reed, J. Chen, A.M. Rawlett, D.W. Price, J.M. Tour, Molecular random access memory cell, Applied Physics Letter 78 (2001) 3735-3737

[5] S.J. Tans, A.R.M. Verschueren, C. Dekker, Room-temperature transistor based on a single carbon nanotube, Nature 393 (1998).

[6] G. Gottarelli, S. Masiero, E. Mezzina, S. Pieraccini, J.P. Rabe, P. Samori, G.P. Spada, The self-assembly of lipophilic guanosine derivatives in solution and on solid surface, Chemical European Journal 6 (2002) 3242-3248.

[7] R. Rinaldi, G. Maruccio, A. Biasco, V. Arima, R. Cingolani, T. Giorgi, S. Masiero, G.P. Spada, G. Gottarelli, Hybrid molecular electronics devices based on a modified deoxy-guanosines, Nanotechnology 13 (2002) 398-403.

[8] G. Maruccio, P. Visconti, S. D’Amico, E. D’Amone, R. Cingolani, R. Rinaldi, Planar nanotips as probes for trans-

port experiments in molecules, Microelectronic Engineering 67-68 (2003) 838-844. 